\title{
CONTRIBUIÇÕES A GESTÃO DA SEGURANÇA E SAUDE OCUPACIONAL DE COLABORADORES DO CULTIVO DO MAMÃO NA REGIÃO DE BARAÚNA-RN
}

\author{
A. C. Barboza Jr', E. A. S. de Medeiros', M. C. de F. Cardoso', M. J. R. Bezerra' e C. E. M. Jerônimo ${ }^{1}$ \\ ${ }^{1}$ Universidade Potiguar \\ aurinojunior15@yahoo.com.br - emilianomedeiros@hotmail.com - marcelo_champa@hotmail.com - \\ melkbarauna@hotmail.com-c_enrique@hotmail.com
}

Artigo submetido em julho de 2012 e aceito em agosto/2013

\section{RESUMO}

As atividades no âmbito agrícola estão ganhando destaque no cenário nacional em se tratando de Segurança do trabalho. Tratar de se conhecer seus perigos é o primeiro passo para ter base em tomadas de decisões e planejamento dessas atividades. Assim sendo, trataremos nesse trabalho, de apontar os possíveis perigos na atividade do cultivo do mamão na Região de Baraúnas no estado do Rio Grande do Norte. Para isso, utilizou-se da ferramenta conhecida como APP (ANÁLISE PRELIMINAR DE PERIGO) para qualificálos mediante tabelas contendo a atividade desempenhada, perigos, causas, modo de detecção, efeito, categorias de frequência, severidade, risco, além de suas recomendações e observações. O levantamento dos dados foi realizado em fazendas produtoras de mamão no município de Baraúna, estado do Rio Grande do Norte. O trabalho foi dividido em etapas de levantamento dos perigos, entrevistas com colaboradores e observação visual das rotinas de trabalho desses. Os resultados apontam para maiores riscos de acidentes com mãos e membros inferiores, tendo-se uma melhor definição dos cenários e quadros de priorização e direcionamentos para uma correta gestão de segurança e saúde dos colaboradores envolvidos nesta atividade.

PALAVRAS-CHAVE: Segurança, Baraúna, mamão, análise, perigo.

\section{CONTRIBUTIONS OF MANAGEMENT OF OCCUPATIONAL SAFETY AND HEALTH EMPLOYEES OF CROP PAPAYA IN THE REGION FROM BARAUNA-RN}

\begin{abstract}
Activities on the farm are gaining prominence on the national stage when it comes to job security. Treating to meet their hazards is the first step to having based decision making and planning of these activities. Therefore, we will in this work, pointing out the possible dangers in the activity of the cultivation of papaya in Region Baraúna in the state of Rio Grande do Norte. For this, we used the tool known as APP (PRELIMINARY ANALYSIS OF DANGER) to qualify them by tables containing the activity performed, dangers, causes, detection mode, and effect, categories of frequency,
\end{abstract}

severity, risk, and its recommendations and observations. The survey was conducted in papaya producing farms in the municipality of Baraúna, state of Rio Grande do Norte. The work was divided into stages lifting hazards, interviews with employees and visual observation of the work of these routines. The results indicate higher risks of accidents with hands and legs, having a better definition of scenarios and prioritization frameworks and guidelines for proper management of safety and health of employees involved in this activity.

KEYWORDS: Security, Baraúnas, papaya, analysis, hazard. 


\section{CONTRIBUIÇÕES A GESTÃO DA SEGURANÇA E SAUDE OCUPACIONAL DE COLABORADORES DO CULTIVO DO MAMÃO NA REGIÃO DE BARAÚNA-RN}

\section{INTRODUÇÃO}

O Carica papaya L. é o mamoeiro mais cultivado comercialmente e também uma das frutíferas tropicais mais difundidas no mundo, produzindo uma fruta de grande aceitação tanto no mercado brasileiro como internacional. Sua cultura, além de grande relevância econômica, desempenha importante função social, gerando grande número de empregos de forma estável, uma vez que produz durante todo o ano e necessita de renovações periódicas, normalmente a cada três ou quatro anos (Leite, 2008).

As regiões produtoras de mamão no mundo estão localizadas em uma faixa do globo terrestre compreendida entre os trópicos de Câncer e Capricórnio a $21^{\circ}$ de latitude Norte e $21^{\circ}$ de latitude Sul. Os países maiores produtores de mamão, considerando a produção de frutos, são Brasil com $27 \%$ do total mundial, seguido por Nigéria (14\%), Índia (12\%), México (11\%), Indonésia (9\%), República Dominicana do Congo (4\%), Peru (3\%), China (2,8\%), Tailândia (2,2\%), Colômbia (2,1\%), conforme descreve Nakamae (2003).

No mamoeiro a sua maior importância econômica está na venda do seu fruto para o consumo ao natural, enviado para os mercados locais e de exportação, como também por possuir fonte importante de papaína, peptina, que são empregadas na indústria têxtil e farmacêutica de alimentos e cosméticos e como características principais, destacam-se as grandes densidades de plantas por hectare, seu rápido desenvolvimento, sua fácil propagação através de sementes, uma alta produtividade e frutos maduros durante todos os meses do ano em clima tropical, com chuvas regulares ou através da irrigação nos locais de pouca chuva ou períodos secos (MANICA et al., 2006).

A agricultura brasileira, e não obstante a cultura do mamoeiro, vem chamando a atenção de especialistas na área de segurança do trabalho, pois todos os dias milhares de trabalhadores estão submetidos a inúmeros riscos na atividade. O trabalho agrícola é uma das que registram os maiores índices de acidentes associados ao trabalho.

Segundo a Organização Internacional do Trabalho - OIT, as atividades laborais que mais matam são: agricultura, mineração, construção e pesca comercial. Por isso foi criada, em 2005, a Norma Regulamentadora de Segurança e Saúde do Trabalho na Agricultura, Pecuária, Silvicultura, Exploração Florestal e Aquicultura - NR-31, do Ministério do Trabalho e Emprego (COUTO, 2007). Dentre os vários riscos ocupacionais, destacam-se os agrotóxicos que são relacionados a intoxicações agudas, doenças crônicas, problemas reprodutivos e danos ambientais (FARIA ET al 2007).

Apesar da grande problemática que envolve os riscos a saúde ocupacional nas atividades agrícolas, poucos são os estudos que auxiliam nas análises e composição de programas que direcionam para políticas e programas adequados de gestão. E conforme se pode observar nos relatos bibliográficos e periódicos especializados existe uma carência de estudos específicos para tal segmento, tendo-se pequenos ensaios relativos a segurança e saúde ocupacional na agricultura em estudos isolados como Almeida (1995), Couto (2007) e Silva et al (2011).

Sendo assim, neste trabalho teve um enfoque voltado para o município de Baraúna-RN, 
segundo IDEMA (2012), localizado na Chapada do Apodi/RN, com uma área de $889 \mathrm{~km}^{2}$, situa-se a uma distância de $312 \mathrm{~km}$ da capital do Estado (Natal) e a $32 \mathrm{~km}$ de Mossoró/RN, centro de referência comercial mais próximo. A escolha da cidade se deu por se tratar de uma área de grande produção de mamão, tendo-se nessa cultura a exigência de cuidados no preparo do solo e a colheita, especialmente, por ter difícil manejo, em função da altura das plantas. Logo, se faz necessário o uso de maquinários durante estas operações, assim aumentando os perigos aos trabalhadores.

No intuito de reduzir tais riscos ambientais, o trabalho apresentará a aplicação de uma metodologia para mapeamento, utilizando a análise preliminar de perigos (APP), e priorização dos riscos inerentes a tal atividade, tendo-se como base fundamental para a elaboração de novos programas de prevenção. O estudo apontará propostas de melhorias para os cenários identificados e mitigação de elementos críticos.

\section{METODOLOGIA}

\section{Espaço Amostral}

A região de estudo se situa no município de Baraúna - $\mathrm{RN}$, mais precisamente no Sítio Sumidouro. É uma empresa na qual se destaca na produção de mamão na cidade, sendo esta produção destinada ao mercado externo como ao também ao mercado interno. A propriedade possui hoje cerca de 135 hectares de mamão plantado, sendo que essas plantações possuem diferentes estágios de desenvolvimento, desde áreas em pleno estágio de colheitas e áreas em estágios de desenvolvimento vegetativo.

O município de Baraúna-RN, segundo dados do senso de 2011, realizado pelo Instituto Brasileiro de Geografia e Estatística (IBGE), sua população é constituída de 24.187 habitantes, sendo que desse total, 6.957 vivem no meio rural, o que correspondem, aproximadamente, a $37 \%$ da população, composta de 3.567 homens e 3.140 mulheres. Tem como atividades econômicas, um modesto comércio, algumas pequenas indústrias, a pecuária e a agricultura (cultivo do mamão, melancia e pequenas propriedades com cultivo para subsistência de milho e feijão). Sendo o cultivo do mamão sua principal fonte de sustentação econômica, cuja maioria das propriedades é de base familiar, que se encontra dividida entre comunidades e assentamentos rurais. Atualmente, é uma região com alto potencial para fruticultura e que apresenta problemas enfrentados pela agricultura mundial, que são os riscos e perigos envolvidos em suas atividades do meio rural. Na Figura 1 apresenta-se o mapa de localização da referida área de estudo.

A área considerada para estudo compreende a utilização de mão de obra pouco qualificada, com grau de instrução inferior ao estudo fundamental (em média, tem-se o quarto ano incompleto). Os empregados são predominantemente de homens (cerca de $90 \%$ ), tendo voltados para atividades práticas da agronomia, em especial, manejo e manipulação dos frutos. A maioria dos empregados não participa de programas de incentivo a continuidade dos estudos de adultos, e estando restritos a experiência profissional na área agrícola. 


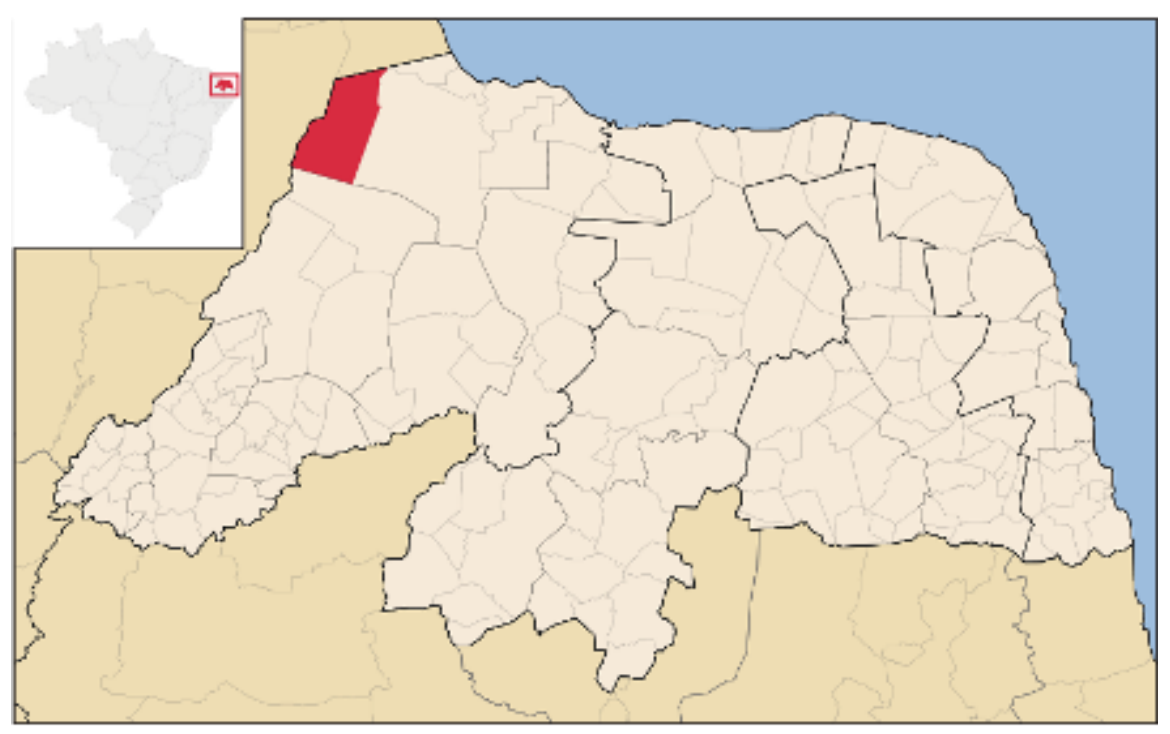

Figura 1 - Área do Município de Baraúna-RN. Fonte: Wikipédia (2012).

\section{Caracterização do Estudo}

O presente artigo tem como objetivo o mapeamento dos riscos ambientais, nos quais os colaboradores da atividade do cultivo do mamão estão expostos na cidade de Baraúnas-RN, por meio da Análise Preliminar de Riscos. Para tanto, foi utilizada a estratégia de pesquisa de natureza qualitativa, classificada também como descritiva, bem como exploratória, e que utilizou para o desenvolvimento de seu processo a técnica do estudo de caso.

A aplicação do presente trabalho pode ser extrapolada para atividades da fruticultura com riscos semelhantes, a saber cultivo da manga, caju e goiaba. Logicamente, atuando com as adaptações que cada processo requer.

O estudo de caso que segundo Yin (2005), representa uma investigação empírica e compreende um método abrangente, com a lógica do planejamento, da coleta e da análise de dados e da escolha do referencial teórico. Pode incluir tanto estudos de caso único quanto de múltiplos, assim como abordagens quantitativas e qualitativas de pesquisa. A metodologia utilizada na elaboração do presente trabalho foi fundamentada na revisão bibliográfica, que envolveu a consulta a: artigos em periódicos científicos e a documentações oficiais (diário oficial, legislações etc). Algumas das informações aqui apresentadas foram extraídas a partir da visita aos websites de universidades brasileiras.

\section{Análise de Risco}

A Análise Preliminar de Perigos (APP) consiste no método indutivo e é estruturada para detectar os potenciais perigos que decorrem da instalação de novas unidades e sistemas desconhecidos ou do próprio funcionamento da planta que opera com materiais perigosos. Este método tem como objetivo analisar as formas pelas quais os componentes do processo podem atuar fora de controle e de forma inesperada, elencando, para cada situação de perigos identificada, as causas, as formas de detecção disponíveis e as consequências sobre os trabalhadores, tanto no entorno na população como sobre o meio ambiente. Em seguida é executada uma avaliação qualitativa dos riscos relacionados, identificando-se, desta maneira, aqueles que necessitam ser priorizados. Além disso, a título de sugestão, incluem-se medidas mitigadoras sobre os riscos, com a finalidade de eliminar os agentes causais ou diminuir as 
consequências nos sistemas com acidentes identificados (ALENCAR, 2009).

A estrutura da APP envolve os eventos considerados perigosos cujas fontes tenham origem no sistema analisado, envolvendo tanto as falhas de materiais ou do sistema, como eventuais erros de operação ou de manutenção (falhas humanas). O resultado final de uma APP consiste na identificação dos perigos e no mapeamento das áreas perigosas com base nos cenários de acidente relativos aos processos, armazenamentos, instalações, transportes de produtos químicos e a outros sistemas inerentes aos processos industriais.

O uso da APP, conforme descreve Camacho (2004), associada com matrizes de aceitabilidade de risco exige que os cenários de acidente sejam classificados em categorias de frequência, as quais fornecem uma indicação qualitativa da frequência esperada de ocorrência para os cenários identificados. Esta escolha dos critérios é função do tipo de análise que se pretende executar, podendo ser definida pela entidade governamental ou pela própria empresa, quando se tratar de estudo interno.

Para o desenvolvimento do trabalho utilizou-se a ferramenta de Análise Preliminar de Perigos (APP), que, segundo Fleming et, al. (1999) e Camacho (2004), permite uma avaliação qualitativa do risco associado a cada um dos cenários identificados, orientando a equipe envolvida na análise para a elaboração de medidas mitigadoras do risco ou, muitas das vezes, para a elaboração de estudos mais aprofundados sobre estes cenários, com a aplicação de técnicas quantitativas como Avaliação de Efeitos Físicos, Análise de Vulnerabilidade e Análise Quantitativa de Riscos. Estas técnicas, por serem baseadas em modelos matemáticos complexos envolvem custos bem maiores, além de exigirem uma quantidade de tempo bem superior para sua aplicação. Assim, torna-se fundamental a otimização do processo de escolha dos cenários que necessitam efetivamente de uma posterior avaliação quantitativa.

Conforme Silva et al.(2011) a Análise Preliminar de Perigo (APP) é uma metodologia fundamentada na identificação dos perigos que podem ser causados através de eventos indesejáveis. A aplicabilidade desta metodologia abrange desde fase de projeto como a de início de operação de um sistema e também na revisão geral de segurança de um sistema já em operação. Ainda ressalta que dentro da APP são apontadas e/ou levantadas as causas de cada um dos possíveis eventos acidentais e as suas respectivas consequências. Em seguida, é feita uma avaliação qualitativa do risco associado a cada cenário acidental, considerando a frequência de ocorrência do evento acidental segundo as causas da severidade do cenário de acidente. Os resultados obtidos configuram-se de forma qualitativa, sem estimativas numéricas.

O levantamento dos dados se deu no processo de preparo do solo, onde é feito utilizando técnicas como gradagem do solo, sulcamento, uso de herbicidas, fertilização etc. Nesta etapa estão envolvidos perigos como atropelamento de funcionário, tombamento do trator, lesões com ferramentas e intoxicações com agrotóxicos.

Outro procedimento alvo do trabalho é a colheita dos frutos. Nesta etapa um trator com uma espécie de plataforma de elevação, deixando o funcionário mais perto dos frutos, facilitando a coleta. Esta fase envolve perigos físicos bastante ocorrentes na atividade e na região, tais como: Queda de funcionário da plataforma de colheita, colisão do maquinário com as plantas, desabamento das caixas de coleta empilhadas e contato do trabalhador com o látex da planta.

Com o levantamento dos possíveis perigos envolvidos na produção de mamão na Região de Baraúna, foi possível realizar o uso da ferramenta APP. Onde são indicados quais os Perigos, sua Causa, Modo de Detecção, Efeito, Categoria de Frequência, Categoria de Severidade, 
Categoria de Risco e suas Recomendações e Observações, divididas em um quadro com as colunas:

- 10 Coluna: Perigo - Coluna que contém os perigos identificados e relacionados a atividade relacionada.

- 2o Coluna: Causa - Nesta coluna são apontadas as possíveis causas de cada perigo. Tais como falhas nos equipamentos como também falhas ou erros de funcionário.

- 3o Coluna: Modo de Detecção - Esta coluna descreve qual modo pode ser indicado para detectar os perigos citados.

- 4 Coluna: Efeitos - Aqui são listados os possíveis prejuízos e consequências geradas a partir dos perigos.

- 5o Coluna: Categoria de Frequência - Já esta coluna utiliza a classificação existente na Tabela 1. Conduzida esta, por histórico de ocorrências de acidentes da região ou também a que se espera ocorrer durante a vida útil do ambiente de trabalho.

- 6o Coluna: Categoria de Severidade - Este item refere-se ao potencial de danos que possa esse perigo, vir a causar ao ambiente, empresa, comunidade e funcionário, contidos na Tabela 2.

- 70 Coluna: Categoria de Risco - Esta é a relação entre as categorias de frequência e severidade obtém-se através da Tabela 3.

- 8o Coluna: Recomendações/ Observações - Já nesta coluna, têm-se recomendações ou observações plausíveis sobre o cenário do acidente.

Para cada um dos perigos associados foram aplicados os cálculos das freqüências da ocorrência, atribuindo uma classificação específica conforme Tabela 1. Da mesma maneira procedeu-se com o enquadramento do grau de severidade, conforme Tabela 2. E por fim, o cruzamento das informações de freqüência e severidade definiu-se o grau de risco dos perigos analisados. Tal procedimento foi baseado no método descrito por Silva (2012).

Tabela 1: Categoria de Freqüência

\begin{tabular}{|c|c|c|}
\hline CATEGORIA & DESCRIÇÃo & PROBABILIDADE \\
\hline $\begin{array}{c}\text { A } \\
\text { Provável }\end{array}$ & $\begin{array}{c}\text { Esperado ocorrer várias vezes } \\
\text { durante a vida útil da } \\
\text { instalação. }\end{array}$ \\
\hline B & $\begin{array}{c}\text { Esperado ocorrer pelo menos } \\
\text { uma vez durante a vida útil da } \\
\text { instalação. }\end{array}$ & $10^{-1}$ \\
\hline Razoavelmente Provável & $\begin{array}{c}\text { Pouco provável de ocorrer } \\
\text { durante a vida útil da } \\
\text { Instalação. }\end{array}$ & $10^{-3} \leq \mathrm{P}<10^{-1}$ \\
\hline Remota & $\begin{array}{c}\text { Teoricamente possível, porém } \\
\text { extremamente pouco } \\
\text { provável de ocorrer durante a } \\
\text { vida útil da instalação. }\end{array}$ & $\mathrm{P}<10^{-3}$ \\
\hline $\begin{array}{c}\text { Extremamente Remota } \\
\text { Fonte: SILVA (2012) }\end{array}$
\end{tabular}

Fonte: SILVA (2012) 
Tabela 2: Categoria de Severidade

\begin{tabular}{|c|c|}
\hline CATEGORIA & DESCRIÇÃO \\
\hline $\begin{array}{c}\text { I } \\
\text { Desprezível }\end{array}$ & Nenhum dano ou dano não mensurável. \\
\hline $\begin{array}{c}\text { II } \\
\text { Marginal }\end{array}$ & Danos irrelevantes ao meio ambiente e às pessoas. \\
\hline III Crítica & $\begin{array}{c}\text { Possíveis danos ao meio ambiente causados por } \\
\text { liberações de substâncias químicas, tóxicas ou } \\
\text { inflamáveis. Pode provocar lesões de gravidade } \\
\text { moderada às pessoas ou impactos ambientais com } \\
\text { tempo reduzido de recuperação. }\end{array}$ \\
\hline IV Catastrófica & $\begin{array}{c}\text { Impactos ambientais devido a liberações de } \\
\text { substâncias químicas, tóxicas, ou inflamáveis. Pode } \\
\text { provocar mortes ou lesões graves às pessoas ou } \\
\text { impactos }\end{array}$ \\
ambientais com tempo de recuperação elevado.
\end{tabular}

Fonte: SILVA,et, al.

Tabela 3: Matriz de Riscos para Priorização

\begin{tabular}{|c|c|c|c|c|}
\hline \multicolumn{2}{|c|}{ SEVERIDADE } & IV Catastrófica \\
\hline Frequência & I Desprezível & $\begin{array}{c}\text { II } \\
\text { Marginal }\end{array}$ & $\begin{array}{c}\text { III } \\
\text { Crítica }\end{array}$ & RA \\
\hline $\begin{array}{c}\text { A } \\
\text { Provável }\end{array}$ & RM & RM & RA \\
\hline $\begin{array}{c}\text { B Razoavelmente } \\
\text { Provável }\end{array}$ & RB & RM & RM \\
\hline $\begin{array}{c}\text { C } \\
\text { Remota }\end{array}$ & RB & RB & RM \\
\hline $\begin{array}{c}\text { D Extremamente } \\
\text { Remota }\end{array}$ & RB & RB & RB & RM \\
\hline
\end{tabular}

Nota: Sendo: RB -Risco Baixo, RM - Risco Médio, RA - Risco Alto.

O desenvolvimento desta técnica pode ser utilizada para o desenvolvimento dos programas de saúde ocupacional recomendados pelo Ministério do Trabalho e do Emprego, mais especificamente na norma regulamentadora de número 9, que estabelece a obrigatoriedade da elaboração e implementação, por parte de todos os empregadores e instituições que admitam trabalhadores como empregados, do Programa de Prevenção de Riscos Ambientais - PPRA, visando à preservação da saúde e da integridade dos trabalhadores, através da antecipação, reconhecimento, avaliação e consequente controle da ocorrência de riscos ambientais existentes ou que venham a existir no ambiente de trabalho, tendo em consideração a proteção do meio ambiente e dos recursos naturais (Brasil, 1994).

\section{RESULTADOS E DISCUSSÕES}

Após os levantamentos e o uso das Tabelas descritas na metodologia, foram desenvolvidos dois quadros respectivamente correspondentes aos perigos ligados ao preparo do solo e à colheita. Na Tabela 4 são apresentados os resultados do mapeamento de riscos para o 
preparo de solo. Nesta observa-se que os riscos de mais críticos estão relacionados a possíveis lesões com ferramentas e a exposição a agentes químicos.

Tabela 4: Resultados para os Riscos Associados ao Preparo do Solo e Plantio

\begin{tabular}{|c|c|c|c|c|c|c|c|c|}
\hline № & PERIGO & CAUSA & $\begin{array}{l}\text { MODO DE } \\
\text { DETECÇÃO }\end{array}$ & EFEITO & $\begin{array}{l}\text { CAT. } \\
\text { FREQ. }\end{array}$ & $\begin{array}{l}\text { CAT. } \\
\text { SEV. }\end{array}$ & $\begin{array}{l}\text { CAT. } \\
\text { RISCO }\end{array}$ & $\begin{array}{l}\text { RECOMENDAÇÕES } \\
\text { E OBSERVAÇÕES }\end{array}$ \\
\hline 1 & $\begin{array}{l}\text { ATROPELAMENTO } \\
\text { DE FUNCIONÁRIO }\end{array}$ & $\begin{array}{c}\text { FALTA DE } \\
\text { ATENÇÃO DO } \\
\text { TRATORISTA, } \\
\text { FALHA MECÂNICA } \\
\text { OU DESATENÇÃO } \\
\text { DO ATROPELADO } \\
\end{array}$ & VISUAL & $\begin{array}{l}\text { FRATURAS, } \\
\text { LUXAÇÕES, } \\
\text { PERDA DE } \\
\text { MEMBROS E } \\
\text { ATÉ MORTE }\end{array}$ & C & IV & RM & $\begin{array}{l}\text { REVISÕES PERIÓDICAS } \\
\text { NO EQUIPAMENTO, } \\
\text { TREINAMENTO DO } \\
\text { TRATORISTA E } \\
\text { SINALIZAR A ÁREA. }\end{array}$ \\
\hline 2 & $\begin{array}{l}\text { TOMBAMENTO } \\
\text { DO TRATOR }\end{array}$ & $\begin{array}{c}\text { DECLIVE } \\
\text { ACENTUADO, } \\
\text { ALTA } \\
\text { VELOCIDADE, } \\
\text { DESATENÇÃO E } \\
\text { CANSAÇO }\end{array}$ & VISUAL & $\begin{array}{l}\text { FRATURAS, } \\
\text { LUXAÇÕES, } \\
\text { PERDA DE } \\
\text { MEMBROS E } \\
\text { ATÉ MORTE }\end{array}$ & D & IV & $\mathrm{RM}$ & $\begin{array}{l}\text { TREINAMENTO PARA O } \\
\text { TRATORISTA E } \\
\text { RESPEITAR OS } \\
\text { HORÁRIOS DE } \\
\text { TRABALHO }\end{array}$ \\
\hline 3 & $\begin{array}{l}\text { LESÕES COM } \\
\text { FERRAMENTAS }\end{array}$ & $\begin{array}{c}\text { FALTA DE } \\
\text { ATENÇÃO, } \\
\text { CANSAÇO, } \\
\text { FADIGA E } \\
\text { INEXPERIÊNCIA }\end{array}$ & VISUAL & $\begin{array}{l}\text { INVALIDEZ } \\
\text { PARCIAL OU } \\
\text { DEFINITIVA }\end{array}$ & A & III & RA & $\begin{array}{l}\text { TREINAMENTO DOS } \\
\text { FUNCIONÁRIOS E USO } \\
\text { DE EPI NECESSÁRIO }\end{array}$ \\
\hline 4 & $\begin{array}{l}\text { INTOXICAÇÃO } \\
\text { COM } \\
\text { AGROTÓXICO }\end{array}$ & $\begin{array}{l}\text { FALTA OU MAU } \\
\text { USO DO EPI, } \\
\text { FALTA DE } \\
\text { TREINAMENTO } \\
\text { OU ERRO NA } \\
\text { DOSAGEM }\end{array}$ & $\begin{array}{c}\text { VISUAL, } \\
\text { ODOR, TATO }\end{array}$ & $\begin{array}{c}\text { DOENÇAS } \\
\text { CRÔNICAS, } \\
\text { DERMATITES, } \\
\text { VÔMITOS E } \\
\text { ATÉ MORTE } \\
\text { QUANDO A } \\
\text { LONGO } \\
\text { PRAZO }\end{array}$ & A & IV & RA & $\begin{array}{c}\text { USO DE EPI, } \\
\text { TREINAMENTO DOS } \\
\text { MANUSEADORES, } \\
\text { CALIBRAÇÃO DOS } \\
\text { PULVERIZADORES. }\end{array}$ \\
\hline
\end{tabular}

$\mathrm{Na}$ Tabela 5 são apresentados os resultados obtidos com o mapeamento dos riscos associados com a colheita dos frutos. Nesse caso, os elementos que requerem maior esforço de gestão são os perigos de queda das plataformas de colheita e desabamento de caixas. Tais elementos apresentam um nível de frequência elevada, conforme observado nas visitas técnicas e acabam por remeter a valores de priorização mais elevados.

Tabela 5: Resultados para os Riscos Associados a Colheita dos Frutos

\begin{tabular}{|c|c|c|c|c|c|c|c|c|}
\hline № & PERIGO & CAUSA & $\begin{array}{l}\text { MODO DE } \\
\text { DETECÇÃO }\end{array}$ & EFEITO & $\begin{array}{l}\text { CAT. } \\
\text { FREQ. }\end{array}$ & $\begin{array}{l}\text { CAT. } \\
\text { SEV. }\end{array}$ & $\begin{array}{l}\text { CAT. } \\
\text { RIS. }\end{array}$ & $\begin{array}{l}\text { RECOMENDAÇÕES } \\
\text { E OBSERVAÇÕES }\end{array}$ \\
\hline 1 & $\begin{array}{l}\text { QUEDA DA } \\
\text { PLATAFORMA } \\
\text { DE COLHEITA }\end{array}$ & $\begin{array}{c}\text { DESATENÇÃO } \\
\text { DO } \\
\text { FUNCIONÁRIO } \\
\text { OU } \\
\text { MOVIMENTO } \\
\text { BRUSCO NO } \\
\text { TRATOR } \\
\end{array}$ & VISUAL & $\begin{array}{l}\text { FRATURAS, } \\
\text { CORTES, } \\
\text { DESMAIOS } \\
\text { OU ATÉ } \\
\text { MORTE }\end{array}$ & B & IV & RA & $\begin{array}{l}\text { NESSÁRIO QUE O COLETADOR } \\
\text { USO CINTO DE PROTEÇÃO }\end{array}$ \\
\hline 2 & $\begin{array}{l}\text { COLISÃO DA } \\
\text { MÁQUINA } \\
\text { COM AS } \\
\text { PLANTAS }\end{array}$ & $\begin{array}{c}\text { DESATENÇÃO } \\
\text { DO TRATORISTA } \\
\text { OU IMPERÍCIA } \\
\text { DO MESMO E } \\
\text { PROBLEMAS } \\
\text { MECÂNICOS }\end{array}$ & VISUAL & $\begin{array}{c}\text { PERDA } \\
\text { PARCIAL } \\
\text { OU TOTAL } \\
\text { DA PLANTA } \\
\text { E DANOS } \\
\text { AO } \\
\text { MAQUINÁR } \\
\text { IO }\end{array}$ & A & II & RM & $\begin{array}{l}\text { TREINAMENTO DO TRATORISTA, } \\
\text { RESPEITO AOS HORÁRIOS DE } \\
\text { TRABALHO E MANUTENÇÃO } \\
\text { PERIÓDICA DO TRATOR }\end{array}$ \\
\hline 3 & $\begin{array}{l}\text { DESABAMENT } \\
\text { O DAS CAIXAS } \\
\text { EMPILHADAS }\end{array}$ & $\begin{array}{c}\text { MAU } \\
\text { ACONDICIONA- } \\
\text { MENTO }\end{array}$ & $\begin{array}{l}\text { VISUALE } \\
\text { MARCAÇÕES } \\
\text { NO CHÃO }\end{array}$ & $\begin{array}{l}\text { FRATURAS } \\
\text { E CORTES }\end{array}$ & A & III & RA & $\begin{array}{l}\text { SUPERVISÃO NO MOMENTO DO } \\
\text { EMPILHAMENTO DAS CAIXAS }\end{array}$ \\
\hline
\end{tabular}




\begin{tabular}{|c|c|c|c|c|c|c|c|c|}
\hline 4 & $\begin{array}{c}\text { CONTATO COM } \\
\text { O LÁTEX DO } \\
\text { MAMÃO }\end{array}$ & $\begin{array}{c}\text { VAZAMENTO } \\
\text { DE LÁTEX DO } \\
\text { FRUTO OU } \\
\text { PLANTA E A } \\
\text { FALTA DO USO } \\
\text { DO EPI }\end{array}$ & $\begin{array}{l}\text { TATO E } \\
\text { VISUAL }\end{array}$ & $\begin{array}{l}\text { QUEIMADU } \\
\text { RAS }\end{array}$ & $A$ & II & $\mathrm{RM}$ & $\begin{array}{c}\text { USO DE LUVAS INDICADAS PARA } \\
\text { A ATIVIDADE }\end{array}$ \\
\hline
\end{tabular}

Nos dois cenários apresentados foram inseridas recomendações para mitigação dos referidos impactos, tendo-se a descrição no item específico das referidas tabelas.

\section{CONCLUSÃO}

Os principais riscos ambientais a segurança e saúde ocupacional dos colaboradores que atuam no processo de cultivo do mamão estão associados: ao uso de agrotóxicos, as lesões com ferramentas, aos riscos de quedas de plataformas de colheita e aos possíveis desmoronamentos de caixas sobre os trabalhadores.

A ferramenta APP demonstrou ser uma ferramenta de fácil manuseio e permitiu conhecer os pontos críticos associados a atividade. Do desdobramento desses resultados surge o potencial de ações preventivas para o emprego na gestão da segurança do trabalho e de seus profissionais no ramo agrícola.

Este trabalho apresenta uma contribuição ao desenvolvimento futuro de programas de prevenção a riscos ambientais presentes na atividade de cultivo do mamão, em especial no padrão adotado no município de Baraúna-RN. Porém, podendo ser extrapolado para cenários do cultivo de culturas como a manga, a goiaba e o caju; também, comum na região nordeste, e que apresentam cenários semelhantes em termos de atividades.

\section{REFERÊNCIAS BIBLIOGRÁFICAS}

1. AGUIAR, L. A. Metodologias de Análise de Riscos - APP \& Hazop. Rio de Janeiro. Disponível em: $\quad$ http://professor.ucg.br/SiteDocente/admin/arquivosUpload/13179/material/APP. Acesso em: 06 jul. 2012.

2. ALENCAR, L. Metodologias de análise de riscos: APP e HAZOP. Rio de Janeiro: UFRJ, 2009, $30 \mathrm{f}$. Disponível em <http://www.saneamento.poli.ufrj.br>. Acesso em 21/06/2012.

3. ALMEIDA, W. F. Trabalho Agrícola e sua Relação com Saúde/Doença. Rio de Janeiro, 1995.

4. ARAÚJO, C. M. De. O Protagonismo das Mulheres Rurais na Produção Orgânica em Baraúna RN. UERN. 2003. Disponível em: http://www.programabolsa.org.br/pbolsa/galeria/arqDownTese/CEDINA_MARIA_DE_ARAUJO.pdf. Acesso em: 11 de Julho de 2012.

5. BRASIL. Nr 9 - Programa De Prevenção De Riscos Ambientais. Portaria SSST n. 25, 29 de dezembro de 1994.

6. CAMACHO, E. N. Uma Proposta de Metodologia para Análise Quantitativa de Riscos Ambientais. (2004). Tese - Programa de Pós-graduação de Engenharia, Universidade Federal do Rio de Janeiro, 2004.

7. CETESB. Manual de orientação para elaboração de estudos de análise de riscos. 2000. 
8. COUTO, J. L. V. do. Segurança no trabalho rural (2007). Disponível em: <http://www.ufrrj.br/institutos/it/de/acidentes/acidente.htm > Acesso em: 2 de julho de 2012.

9. FARIA, N. M. X.; FASSA, A. C. G.; FACCHINI, L. A. Intoxicação por agrotóxicos no Brasil: os sistemas oficiais de informação e desafios para realização de estudos epidemiológicos. Ciênc. Saúde coletiva, Rio de Janeiro, v. 12, n. 1, 2007 . Disponível em: http://www.scielo.br/scielo.php? script=sci_arttext \&pid=S1413$81232007000100008 \&$ Ing=pt\&nrm=iso Acesso em: 10 de Julho de 2012.

10. FLEMING, P. V.; GARCIA, C. De B. Avaliação de riscos industriais e ambientais com a análise preliminar de perigos (APP) e lógica Fuzzy. UNIFACS. PRINCIPIA. Salvador-BA. 2012.

11. IDEMA. Baraúnas. Disponível em: http://www.idema.rn.gov.br. Acesso em: 15/07/2012.

12. LEITE, K. C. O uso de agrotóxicos pelos trabalhadores rurais do assentamento catingueira Baraúna-RN. Revista Verde (Mossoró - RN - Brasil) v.3, n.4, p. 06-28 de outubro/dezembro de 2008.

13. MANICA, I.; MARTINS, D. dos S.; VENTURA, J. A. Mamão: Tecnologia de produção, póscolheita, exportação, mercados. Porto Alegre, RS: Cinco Continentes, 2006. 361 p.

14. NAKAMAE, I. J. ed. Anuário da Agricultura Brasileira. São Paulo: Editora Argos Comunicação, 2003. p.378-386.

15. SILVA, A. C. B. da; et al. Operação Offloading: Análise Preliminar de Perigo e os Impactos Ambientais. Revista Eletrônica - Novo Enfoque, ano 2011, v. 13, n. 13, p. 207 - 221. Disponível em: http://www.castelobranco.br/sistema/novoenfoque/files/13/artigos/18 Aline_ Outros_ProfVilma_Outros_VF.pdf. Acesso em: 11 de Julho de 2012.

16. SILVA, J. R. et al. Protocolo de ações preventivas aos riscos ocupacionais presentes em indústrias de produção sucroalcooleira. Revista Eletrônica de Enfermagem do Centro de Estudos de Enfermagem e Nutrição [serial on-line] 2011 ago-set 2(2):1-16. Available from: <http://www.ceen.com.br/revistaeletronica>. 